\section{Grasping the Hedgehog}

\section{By Lev Osherovich, Senior Writer}

Although at least five companies are developing therapeutics that block the Hedgehog signaling pathway to treat solid tumors, the inhibitor approach thus far has focused on a single target-Smoothened. Now, a trio of publications points to new places to hit the pathway and implicates Hedgehog in hematological cancers.

A paper in Nature opens up a Hedgehog front in the hematological cancer space by showing its role in chronic myelogenous leukemia (CML). ${ }^{1}$ Meanwhile, two papers, one in Nature Chemical Biology ${ }^{2}$ and one in the Proceedings of the National Academy of Sciences, ${ }^{3}$ point to new Hedgehog targets (see Box 1, "Hedgehogomirs").

In mammals, Hedgehog signaling starts with the binding of an extracellular protein called Sonic hedgehog homolog (SHH) to a surface receptor called Patched (PTCH1). This binding inhibits PTCH1, leading to activation of a surface receptor called Smoothened homolog ${ }^{4}$ (SMO; see Figure 1, "Hitting the Hedgehog pathway in cancer").

In normal embryos, SMO activation leads to the expression of genes needed for stem cell maintenance via the transcription factors Glioma-associated oncogene homolog 1 (GLI1) and GLI2. However, inappropriate activation of GLI1 and GLI2 in adult cells leads to the development of cancer stem cells that feed tumor growth by producing an endless stream of transformed cells.

Curis Inc. and partner Genentech Inc. have the most advanced SMO inhibitor, GDC-0449, in Phase II trials for solid tumors. Infinity Pharmaceuticals Inc. is running a Phase I trial of its IPI-926 to treat metastatic and advanced solid tumors. BMS-833923 (XL139) from partners Exelixis Inc. and Bristol-Myers Squibb Co. is in Phase I testing for advanced solid tumors.

Philip Beachy, professor of developmental biology at Stanford University and a coauthor of the Nature paper, told SciBX that the Hedgehog pathway is one of several responsible for the self-renewing properties of many aggressive tumors.

Typical cancer-causing mutations, such as those in receptor tyrosine kinases and tumor suppressors, promote uncontrolled growth. However, abnormal activation of Hedgehog in adult cells reawakens a genetic program of self-renewal normally found in embryonic stem cells.

"Activating these pathways may be the most comprehensive one-stop method for a wannabe cancer to lock in self-renewal," said Beachy.

Cancer therapeutics that kill highly proliferative cells don't necessarily target the slow-growing, self-renewing cancer stem cells responsible for replenishing the tumor stock. ${ }^{5}$

\section{Stemming the stem cells}

The results published in Nature bolster the case for a new company in the Hedgehog space-Fate Therapeutics Inc., which was cofounded by Beachy. The biotech has a preclinical compound for CML that targets an undisclosed Hedgehog pathway component downstream of SMO.

Fate's lead compound is FT1050, a small molecule that stimulates hematopoietic stem cell engraftment and is in Phase I trials for bone marrow transplantation.

"A significant amount of research has focused on Hedgehog signaling in solid tumors such as medulloblastoma and basal cell carcinoma," said Tannishtha Reya, lead author of the Nature article. "But the role of Hedgehog in hematologic malignancies is just beginning to be appreciated." Reya is an associate professor of pharmacology and cancer biology at Duke University.

CML is usually treated with Gleevec imatinib, a BCR-ABL tyrosine kinase inhibitor marketed by Novartis AG. However, the kinase inhibitor doesn't completely eliminate CML tumor cells, and Gleevec-resistant cells can eventually arise.

Targeting the Hedgehog pathway could potentially eliminate the self-renewing stem cell-like progenitor cells that drive CML via chronic activation of Hedgehog signaling.

Reya and colleagues first suspected the Hedgehog-CML connection when they made knockouts of the mouse version of SMO in hematopoietic stem cells and found that these white blood cell precursors were unable to regenerate properly. Because regeneration is a characteristic of both normal stem cells and cancer stem cells, Reya's team tested the effect of SMO deletion in a mouse model of CML. They found that hematopoietic stem cell-specific deletion of Smo decreased tumor cell growth and improved survival compared with what was seen in wild-type controls.

"About half the mice didn't get the disease, and the other half of the mice had a significant delay in disease progression," said Reya.

Reyas team obtained similar results with two small molecule SMO inhibitors-cyclopamine, a teratogenic plant-derived compound used in the laboratory, and an undisclosed preclinical compound from Pfizer Inc. Both were effective in Gleevec-resistant cell lines.

Conversely, overexpressing an activated form of Smo increased the number of CML-associated stem cells and lowered mouse survival compared with what was seen in mock-treated controls.

Reya thinks that inhibiting SMO is ready for clinical testing in CML and is hoping to design a combination therapy trial with SMO antagonists and Gleevec. She also wants to test the effect of inhibiting SMO in other leukemias.

Duke and Stanford have filed for a patent based on the work, and the IP is available for licensing.

\section{Hedging against Hedgehog}

Screens for Hedgehog pathway antagonists have almost always converged on SMO, according to Beachy. One reason is that SMO is a relatively druggable seven-spanning $G$ protein-coupled receptor, whereas other components in the pathway can be more structurally complex and inaccessible. 


\section{Box 1. Hedgehogomirs}

\section{A study by St. Jude Children's}

Research Hospital researchers suggests another angle of attack on the Hedgehog pathway by showing that a cluster of microRNAs contribute to the cancer pathway's activity in medulloblastoma.

miRNAs are small, noncoding, regulatory transcripts that help shape a variety of developmental patternings.

The molecules are being pursued as targets or therapeutics in cardiovascular and certain oncology indications, but their role in medulloblastoma, a common form of childhood brain tumor, had not previously been suspected, according to Martine Roussel, a member in the Department of Tumor Cell Biology and Genetics at St. Jude.

Her team hypothesized that miRNAs involved in early brain development might become abnormally reactivated in Hedgehog-driven tumors.

To test the hypothesis, Roussel collaborated with Greg Hannon of Cold Spring Harbor Laboratory to catalog the miRNAs involved in brain development and to look for their presence in medulloblastomas. Hannon, a professor at Cold Spring Harbor, is also an investigator at the Howard Hughes Medical Institute.

In a paper in the Proceedings of the National Academy of Sciences, ${ }^{3}$ the team reported finding a cluster of seven miRNAs, collectively termed Oncomir-1, that was overexpressed in mouse and human medulloblastomas compared with that seen in healthy tissue. In murine granule neuron progenitor cells with partially compromised Hedgehog pathway mutations, overexpression of Oncomir-1 led to medulloblastoma.

"I don't see any reason why this couldn't be a mechanism that at least contributes to tumor formation," said Philip Beachy, professor of developmental biology at Stanford University.

Roussel said Oncomir-1's mechanism of action is still unknown. It's also unclear whether Oncomir-1 or other miRNAs play a role in Hedgehog-driven tumors in other tissues. However, because miRNAs typically act in the nucleus to modulate transcription and RNA processing, one possibility is that Oncomir-1 affects the genes activated by Glioma-associated oncogene homolog 1 (GLI1) and GLI2, a pair of transcription factors that sit at the bottom of the Hedgehog pathway (see Figure 1, "Hitting the Hedgehog pathway in cancer").

Roussel's next step is to prove that knocking down Oncomir-1 could have a beneficial effect. She is collaborating with Santaris Pharma A/S to antagonize Oncomir-1 using the company's locked nucleic acid (LNA) technology. LNAs are synthetic, degradationresistant RNA analogs that Santaris said have high binding affinity to com- plementary RNA and improved tissue uptake in vivo compared with conventional small interfering RNA.

Sakari Kauppinen, director of miRNA research at Santaris, told SciBX that the company is collaborating with Roussel to explore the functional interactions between $\mathrm{SHH}$ and Oncomir-1 using LNAs. The findings by Roussel suggest that "targeting of Oncomir-1 could provide a new therapeutic approach for treatment of medulloblastoma patients with activated $\mathrm{SHH}$ pathway," he said.

Ideally, knocking down Oncomir-1 in mouse medulloblastomas would complement other strategies for hitting the Hedgehog pathway and slow down tumor growth. However, Beachy was skeptical about the prospects for siRNA delivery to the cerebellum, the brain region affected by medulloblastoma, because breaching the blood brain barrier with nucleic acid therapeutics is a challenge.

"To kill a cancer, you've got to hit all of the cells, so I don't know if you can do this with siRNAs," he said.

Roussel is also performing a prospective study of medulloblastoma patients to determine whether Oncomir-1 expression profiling could be a useful diagnostic for identifying medulloblastoma subtypes.

The findings have not been patented. $-L Z O$
"Smoothened is the proverbial broad side of the barn," he said.

Now, a team at Massachusetts General Hospital (MGH) and the Broad Institute of Massachusetts Institute of Technology and Harvard has developed a small molecule inhibitor of SHH itself.

The molecule, called robotnikinin, was
"This is the first reported small molecule in the literature that binds to Sonic hedgehog." - Lee Peng,

Massachusetts General Hospital
Robotnikinin was identified in a screen for small molecules that bound to mouse and human versions of $\mathrm{SHH}$ in vitro. The compound blocked transcription of the Hedgehog pathway targets in primary human keratinocytes treated with SHH compared with that seen in mocktreated controls.

Robotnikinin did not prevent transcription described in Nature Chemical Biology by a team led by Lee Peng, a physician at MGH, and Stuart Schreiber, director of chemical biology at the Broad Institute.

"This is the first reported small molecule in the literature that binds to Sonic hedgehog," said Peng. triggered by small molecule agonists of SMO, suggesting that the compound acts earlier in the pathway than SMO.

Peng suspects that robotnikinin interferes with SHH's ability to bind PTCH1, but he said elucidating the precise mechanism will require further structural studies. 
Figure 1. Hitting the Hedgehog pathway in cancer. Three studies suggest new ways to target the Hedgehog pathway in cancer. In humans, the extracellular protein Sonic hedgehog homolog $(\mathrm{SHH})$ binds to and blocks Patched (PTCH1), a transmembrane receptor [a], which relieves the inhibition [b] of another transmembrane protein, Smoothened (SMO). SMO goes on to activate glioma-associated oncogene homolog 1 (GLI1) and GLI2 [c], which are transcription factors that travel into the nucleus to activate the expression of tumor-promoting genes [d].

Zhao et al. found that activation of this pathway promotes chronic myelogenous leukemia $(\mathrm{CML})$ in mice.

Separately, Stanton et al. identified a small molecule antagonist of $\mathrm{SHH}$ called robotnikinin, which the researchers suspect interferes with SHH's ability to bind PTCH1.

Uziel et al. found that a cluster of micro-

RNAs collectively called Oncomir-1 promotes Hedgehog pathway activity, perhaps by modulating the activity of GLI1 and GLI2 inside the nucleus [e]. The researchers are now working with Santaris Pharma A/S to develop antagomirs, which are degradation-resistant small interfering RNA, to antagonize Oncomir-1.

A number of companies are developing antagonists of SMO. Additionally, Fate Therapeutics Inc. has a preclinical compound for CML that targets an undisclosed Hedgehog signaling pathway component downstream of SMO.

Beachy said Peng's study was a promising first step toward targeting a different player in the Hedgehog pathway than SMO. However, he questioned whether robotnikinin would be effective against $\mathrm{SHH}$ in vivo. In animals, $\mathrm{SHH}$ is covalently modified with cholesterol and palmitate and is packaged into lipoprotein particles, a process Beachy suggested could render the target inaccessible.

Jeffrey Tong, VP of corporate and product development at Infinity, also has doubts about $\mathrm{SHH}$ as a drug target. He said a small molecule SHH inhibitor would need to be extremely potent because of SHH's relatively high abundance and wide distribution compared with immobile surface receptors such as SMO.

"It will be important to further engineer robotnikinin for tight binding and a slow dissociation rate from the Hedgehog ligand," said Tong. Peng agreed that robotnikinin must prove itself in ongoing mouse tumor studies before it can move toward the clinic. The compound is the subject of a pending patent by Harvard University and is available for licensing.

Osherovich, L. SciBX 2(6); doi:10.1038/scibx.2009.212

Published online Feb. 12, 2009

\section{REFERENCES}

1. Zhao, et al. Nature; published online Jan. 18, 2009;

doi:10.1038/nature07737

Contact: Tannishtha Reya, Duke University, Durham, N.C.

e-mail: t.reya@duke.edu
2. Stanton, B.Z. et al. Nat. Chem. Biol.; published online Jan. 18, 2009; doi:10.1038/nchembio.142

Contact: Stuart Schreiber, Harvard University, Cambridge, Mass.

e-mail: stuart_schreiber@harvard.edu

3. Uziel, T. et al. Proc. Natl. Acad. Sci. USA; published online Feb. 2, 2009; doi:10.1073/pnas.0809579106

Contact: Martine F. Roussel, St. Jude Children's Research Hospital, Memphis, Tenn.

e-mail: martine.roussel@stjude.org

4. Rubin, L.L. \& de Sauvage, F.J. Nat. Rev. Drug Discov. 5, 1026-1033 (2006)

5. Osherovich, L. SciBX 2(4); doi:10.1038/scibx.2009.125

\section{COMPANIES AND INSTITUTIONS MENTIONED}

Broad Institute of Massachusetts Institute of Technology and Harvard, Cambridge, Mass.

Bristol-Myers Squibb Co. (NYSE:BMY), New York, N.Y. Cold Spring Harbor Laboratory, Cold Spring Harbor, N.Y. Curis Inc. (NASDAQ:CRIS), Cambridge, Mass.

Duke University, Durham, N.C.

Exelixis Inc. (NASDAQ:EXEL), South San Francisco, Calif.

Fate Therapeutics Inc., Seattle, Wash.

Genentech Inc. (NYSE:DNA), South San Francisco, Calif.

Harvard University, Cambridge, Mass.

Howard Hughes Medical Institute, Chevy Chase, Md.

Infinity Pharmaceuticals Inc. (NASDAQ:INFI), Cambridge, Mass.

Massachusetts General Hospital, Boston, Mass.

Novartis AG (NYSE:NVS; SWX:NOVN), Basel, Switzerland

Pfizer Inc. (NYSE:PFE), New York, N.Y.

Santaris Pharma A/S, Horsholm, Denmark

St. Jude Children's Research Hospital, Memphis, Tenn.

Stanford University, Stanford, Calif. 